\title{
A phase I study of S-1 in combination with $n a b$-paclitaxel in patients with unresectable or recurrent gastric cancer
}

\author{
Norisuke Nakayama $^{1} \cdot K_{\text {Kenji Ishido }}^{2} \cdot K^{3}$ isho Chin $^{3} \cdot K_{\text {Ken Nishimura }}{ }^{1}$. \\ Mizutomo Azuma $^{2} \cdot$ Satoshi Matsusaka ${ }^{3}$ ' Yasuhiro Inokuchi ${ }^{1} \cdot$ Satoshi Tanabe $^{2}$. \\ Yosuke Kumekawa $^{3} \cdot$ Wasaburo Koizumi ${ }^{2}$
}

Received: 12 January 2016/Accepted: 1 May 2016/Published online: 17 May 2016

(C) The International Gastric Cancer Association and The Japanese Gastric Cancer Association 2016

\begin{abstract}
Background In Japan, S-1, an oral fluoropyrimidine, plus cisplatin is a standard regimen for advanced gastric cancer, whereas $n a b$-paclitaxel is a treatment option. We aimed to evaluate the tolerance, pharmacokinetics, safety, and clinical efficacy of S-1 combined with nab-paclitaxel in patients with advanced gastric cancer in a phase 1 study. Methods The primary objective was to determine the maximum tolerated dose (MTD) and recommended dose (RD) of S-1 plus nab-paclitaxel. The study was designed in accordance with a standard $3+3$ method. Patients received 3-week cycles of treatment. S-1 was administered orally at $80 \mathrm{mg} / \mathrm{m}^{2}$ twice daily for 14 days, and $n a b$-paclitaxel was administered as an intravenous infusion at 180 , 220 , or $260 \mathrm{mg} / \mathrm{m}^{2}$ on day 1 or 8 .

Results Among the 16 patients enrolled, dose-limiting toxicity was observed in one patient at level 2a (S-1 $80 \mathrm{mg} /$ $\mathrm{m}^{2}$ twice daily plus $n a b$-paclitaxel $220 \mathrm{mg} / \mathrm{m}^{2}$ on day 1 ). The MTD was not obtained, but the RD was established as level 3a (S-1 $80 \mathrm{mg} / \mathrm{m}^{2}$ twice daily plus $n a b$-paclitaxel $260 \mathrm{mg} / \mathrm{m}^{2}$ on day 1$)$. The most common grade $3-4$ toxicity was neutropenia $(62.5 \%)$. The overall response rate
\end{abstract}

Trial Registration ID: JapicCTI-121987.

Norisuke Nakayama

norisuke@kcch.jp

1 Department of Gastroenterology, Kanagawa Cancer Center, 2-3-2 Nakao, Asahi-ku, Yokohama, Kanagawa 241-0815, Japan

2 Department of Gastroenterology, Kitasato University School of Medicine, Kanagawa, Japan

3 Department of Gastroenterology, Cancer Institute Hospital, Tokyo, Japan was $54.5 \%$. The pharmacokinetic profiles of coadministered S-1 and paclitaxel were comparable to those of $n a b-$ paclitaxel or S-1 alone.

Conclusions Based on the present results, the RD was determined as level 3 a (S-1 $80 \mathrm{mg} / \mathrm{m}^{2}$ twice daily plus $n a b$ paclitaxel $260 \mathrm{mg} / \mathrm{m}^{2}$ on day 1). This combination therapy was well tolerated and showed antitumor efficacy in patients with advanced gastric cancer.

Keywords S-1 $\cdot N a b$-paclitaxel $\cdot$ Gastric cancer

\section{Introduction}

Gastric cancer is the third leading cause of cancer deaths worldwide and the second leading cause in Japan. Although the prevalence of gastric cancer is tending to decrease around the world, it continues to increase in Japan because of the aging population [1].

Although advanced gastric cancer is generally treated with both surgery and chemotherapy, advanced unresectable or recurrent gastric cancer (AGC) is treated with chemotherapy alone. Chemotherapy for advanced unresectable or recurrent gastric cancer has become highly cytoreductive based on remarkable progress in treatment, but remains insufficiently effective to result in a complete cure and has some unsatisfactory outcomes, thus requiring the development of new treatment strategies.

In Japan, clinical studies have been conducted with a focus on 5-fluorouracil (5-FU) for many years. In the JCOG9205 trial [2], 5-FU/cisplatin (CDDP) combination therapy did not provide statistically significant survival benefits compared with 5-FU alone when given as a continuous intravenous infusion. Therefore, 5-FU monotherapy has been recommended in Japan since that time. 
$\mathrm{S}-1$, an oral fluoropyrimidine, is based on biochemical modulation of 5-FU. S-1 is designed to maintain high levels of 5-FU in plasma and tumor cells with reduced gastrointestinal toxicity by combining tegafur (FT), a prodrug of 5-FU, with the biomodulators 5-chloro-2,4-dihydroxypyridine (CDHP) and oteracil potassium (Oxo) [3].

Two phase III studies on use of S-1 for AGC have been reported. As the JCOG9912 trial [4] demonstrated noninferiority of S-1 to 5-FU in terms of overall survival (OS) and the SPIRITS trial [5] demonstrated superiority of S-1/ CDDP combination therapy (SP therapy) to $\mathrm{S}-1$ alone in terms of OS, SP therapy has been established as a standard therapy for gastric cancer in Japan, and clinical development has focused on S-1.

Given the report by Nukatsuka et al. [6] that the combination of paclitaxel and S-1 resulted in a synergistic reduction in tumor mass in a mouse model of human breast cancer, clinical studies of combination therapy with paclitaxel and S-1 for AGC have been conducted in anticipation of synergy between these two drugs. This combination therapy was shown to be highly effective, with response rates ranging from $50 \%$ to $60 \%$ in clinical studies [7-9].

Paclitaxel, which is insoluble in water and therefore had been dissolved in Cremophor EL (polyoxyethylene castor oil) and anhydrous ethanol, was often associated with side effects related to these vehicles. Nanoparticle albuminbound paclitaxel (nab-paclitaxel) was created to resolve these vehicle-related problems by binding paclitaxel to albumin and thereby making it soluble in physiological saline. Nab-paclitaxel has been clinically developed for breast cancer, non-small cell lung cancer, and pancreatic cancer, among others. It has also been clinically developed for gastric cancer in Japan, and the results of a phase II study have been published [10]. In the study, it was found that $n a b$-paclitaxel given at a dose of $260 \mathrm{mg} / \mathrm{m}^{2}$ every 3 weeks as a second-line treatment for previously 5-FUtreated AGC resulted in a response rate of $27.6 \%$ and a median survival of 9.2 months.

Nab-paclitaxel and S-1 damage cells through different mechanisms of actions. The main toxicity shared by nabpaclitaxel and S-1 is myelosuppression, with no other common toxicological profiles that may affect the continuation of treatment. In addition, the results of nonclinical studies indicated a high efficacy of this combination therapy arising through synergism, leading to our decision to conduct a study of S-1 combined with $n a b$-paclitaxel in patients with AGC [6]. In the present phase I study, we aimed to evaluate the tolerance, pharmacokinetics, safety, and clinical efficacy of S-1 combined with $n a b$-paclitaxel in patients with unresectable or recurrent gastric cancer.

\section{Patients and methods}

This study was conducted in accordance with the Good Clinical Practice guidelines and the Helsinki Declaration, and the protocol was approved by the Institutional Review Board of each participating medical institution before initiation of the study. Written informed consent was obtained from all patients before participation in the study.

\section{Study objectives}

The primary objective of the study was to determine the maximum tolerated dose (MTD) of nab-paclitaxel and the recommended dose (RD) of S-1/nab-paclitaxel combination therapy in patients with AGC. The secondary objectives were to evaluate the safety and efficacy of S-1/nabpaclitaxel combination therapy and to evaluate the pharmacokinetics.

\section{Patients}

Patients with AGC who met all the following criteria were eligible to participate in the study: unresectable or recurrent gastric cancer histologically or cytologically diagnosed as adenocarcinoma; evaluable lesion(s) identified by imaging (e.g., CT), with or without measurable lesion(s) defined by the Response Evaluation Criteria in Solid Tumors (RECIST) v1.1; previously untreated with chemotherapy for AGC, except for preoperative or postoperative adjuvant chemotherapy, which was allowed for patients with recurrent gastric cancer who had evidence of recurrence on images 180 days or more after the completion of chemotherapy; Eastern Cooperative Oncology Group performance status of $0-1$; and neutrophil count $\geq 2,000 / \mathrm{mm}^{3}$, hemoglobin $\geq 8.0 \mathrm{~g} / \mathrm{dl}$, platelet count $\geq 100,000 / \mathrm{mm}^{3}$, serum total bilirubin $\leq 1.5$ times upper limit of normal (ULN), creatinine clearance $\geq 60 \mathrm{ml} / \mathrm{min}$, and aspartate aminotransferase, alanine aminotransferase, and alkaline phosphatase levels $\leq 2.5$ times ULN. Patients who met any of the following criteria were excluded from the study: peripheral sensory neuropathy or peripheral motor neuropathy. HER2 status was not included in the eligibility criteria.

\section{Study design}

This was a phase I, multicenter, open-label, $3+3$ design, dose-escalation study, conducted at three institutions in Japan. Three patients were enrolled at each level. If doselimiting toxicity (DLT) occurred in one of the three patients, an additional three patients were enrolled. The dosage schedules are shown in Fig. 1, and the dosages at 
Schedule A

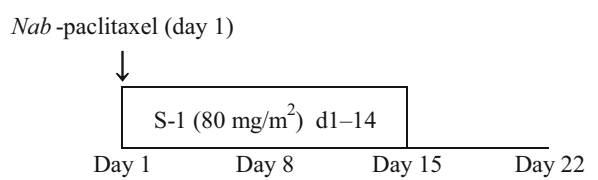

Schedule B

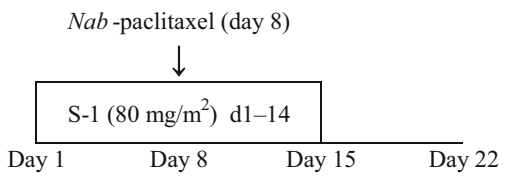

Fig. 1 Therapeutic experimental regimens for the combination of S-1 and $n a b$-paclitaxel

Table 1 Dose levels and schedules

\begin{tabular}{lll}
\hline Dose level & $N a b$-paclitaxel $\left(\mathrm{mg} / \mathrm{m}^{2}\right)$ & $\mathrm{S}-1\left(\mathrm{mg} / \mathrm{m}^{2}\right)$ \\
\hline Schedule A & & 80 \\
Level 1a & 180 & \\
Level 2a & 220 & \\
Level 3a & 260 & 80 \\
Schedule B & & \\
Level 1b & 180 & \\
Level 2b & 220 & \\
Level 3b & 260 & \\
\hline
\end{tabular}

each level are shown in Table 1. If one or fewer of three patients experienced DLT at level $3 \mathrm{a}$ or $3 \mathrm{~b}$, an additional three patients were enrolled and a total of six patients were evaluated. The dose level of $\mathrm{S}-1$ was $80 \mathrm{mg} / \mathrm{m}^{2}$ based on body surface area (BSA) and administered orally at a dose of $40 \mathrm{mg} / \mathrm{m}^{2}$ twice daily for 14 consecutive days, followed by a 7-day washout. $\mathrm{Nab}$-paclitaxel was administered at three dose levels of 180,220, and $260 \mathrm{mg} / \mathrm{m}^{2}$ (level 1, level 2 , and level 3, respectively) based on the BSA, and administered as an intravenous infusion over $30 \mathrm{~min}$ on day 1 or 8 . Administration of $n a b$-paclitaxel was performed according to two dosage schedules: schedule A (administration on day 1) and schedule B (administration on day 8); this was based on the assumption that neutropenia would frequently occur at approximately 8 days after administration of $n a b$-paclitaxel. In other words, if $n a b$ paclitaxel was administered on day 8, nab-paclitaxel-related myelosuppression would occur on day 15 , when S-1 was not administered for washout, resulting in an increased probability of continued S-1 therapy and allowing an exploration of the dosage schedule for $n a b$-paclitaxel. The dose levels were changed as shown in Fig. 2. Each cycle could be started if all the following criteria were met: neutrophil count $\geq 1,500 / \mathrm{mm}^{3}$; platelet count $\geq 75,000 /$ $\mathrm{mm}^{3}$; and grade 2 or less peripheral sensory neuropathy. S-1 was suspended if any of the following criteria were met during any cycle: neutrophil count $<1,000 / \mathrm{mm}^{3}$; platelet count $<50,000 / \mathrm{mm}^{3}$; and grade 3 or higher diarrhea or oral mucositis. The criteria for dose reduction were as follows: both $\mathrm{S}-1$ and $n a b$-paclitaxel were reduced if neutrophil count $<500 / \mathrm{mm}^{3}$ persisted for at least 3 days, febrile neutropenia occurred, or platelet count decreased to $50,000 / \mathrm{mm}^{3}$ or less; only nab-paclitaxel was reduced if grade 3 peripheral sensory neuropathy occurred; and only $\mathrm{S}-1$ was reduced if grade 3 diarrhea or oral mucositis occurred.

\section{Definition of dose-limiting toxicity}

The severity of treatment-related adverse events was evaluated in accordance with the Common Terminology Criteria for Adverse Events (CTCAE) Version 4.0, and DLT was defined as any of the following treatment-related adverse events observed in the first cycle: grade 4 neutropenia that persisted for at least 3 days; febrile neutropenia; other grade 4 hematotoxicity; grade 3 or higher nonhematological toxicity; treatment-related adverse event leading to a delay of 8 days or more in the start of the second cycle; and treatment-related adverse event leading to skipping of the $n a b$-paclitaxel dose on day 8 specified in schedule B.

\section{Estimation of MTD and determination of RD}

The MTD was defined as the dose level at which at least two of three patients or at least two of six patients experienced DLT. In principle, the RD was defined as the dose level immediately below the estimated MTD.

Fig. 2 Dose escalation schema (DLT dose-limiting toxicity)

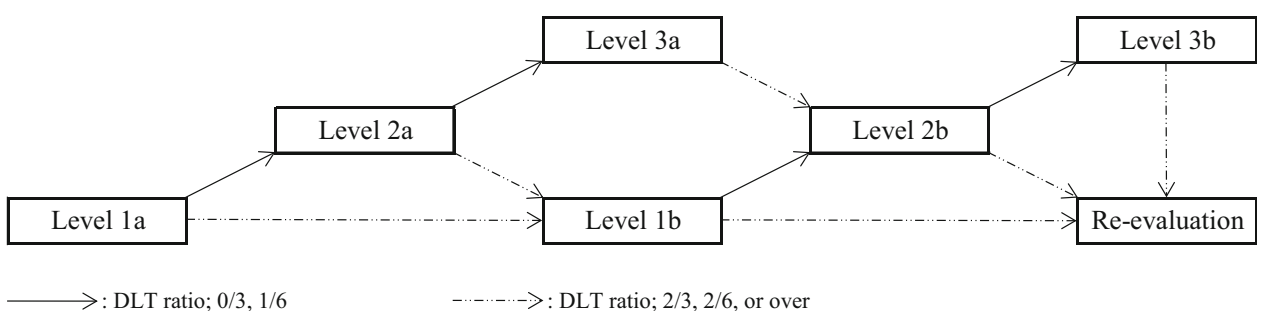




\section{Assessments}

The severity of adverse events was evaluated in accordance with the CTCAE Version 4.0. The antitumor response was evaluated based on imaging every two cycles in accordance with RECIST v1.1.

\section{Pharmacokinetics}

Pharmacokinetics was evaluated in three patients of each level. The observed plasma concentrations of FT, 5-FU, CDHP, Oxo, and paclitaxel were used to calculate the pharmacokinetic parameters. Plasma samples were collected during the first cycle only. At the dose levels in schedule A (levels 1a, 2a, and 3a), samples were collected at $0,0.5,1,2,4,6,8,12,24,48$, and $72 \mathrm{~h}$ after administration of $n a b$-paclitaxel (nab-PTX) on day 1 . At the dose levels in schedule B (levels $1 \mathrm{~b}, 2 \mathrm{~b}$, and $3 \mathrm{~b}$ ), samples were collected at $0,0.5,1,2,4,12,24,48$, and $72 \mathrm{~h}$ after administration of $n a b$-PTX on day 8 . The plasma concentrations of FT, 5-FU, CDHP, Oxo, and paclitaxel were measured by Shin Nippon Biochemical Laboratories (Wakayama, Japan) using validated methods of high-performance liquid chromatography with tandem mass spectrometry (LC/MS/MS). The lower limits of quantification were 20, 2, 4, 4, and $1 \mathrm{ng} / \mathrm{ml}$ for FT, 5-FU, CDHP, Oxo, and paclitaxel in human plasma, respectively. The pharmacokinetic parameters were separately calculated from the individual plasma concentrations of each compound using a noncompartment analysis program within the pharmacokinetic analysis software package Phoenix WinNonlin (Pharsight, Mountain View, CA, USA).

\section{Results}

\section{Patient characteristics}

A total of 16 patients were enrolled in the study between August 2011 and July 2013, and followed up until September 2014. All patients received at least one cycle of study treatment and were included in the safety and efficacy analyses. Among the 16 patients, 1 patient at level $3 \mathrm{a}$ who discontinued use of $\mathrm{S}-1$ without permission was excluded from the DLT evaluation because of poor treatment compliance. The patient characteristics are summarized in Table 2 . The 16 patients comprised 12 males and 4 females, of whom 1 had postoperative recurrent gastric cancer and 15 had unresectable gastric cancer; 14 patients had nodal metastasis, and 7 had peritoneal metastasis.
Table 2 Patient characteristics

\begin{tabular}{|c|c|c|c|c|}
\hline$n$ & $\begin{array}{l}\text { Level 1a } \\
3\end{array}$ & $\begin{array}{l}\text { Level 2a } \\
6\end{array}$ & $\begin{array}{l}\text { Level 3a } \\
7\end{array}$ & $\begin{array}{l}\text { Total }(\%) \\
16\end{array}$ \\
\hline \multicolumn{5}{|l|}{ Gender } \\
\hline Male & 3 & 4 & 5 & $12(75.0)$ \\
\hline Female & 0 & 2 & 2 & $4(25.0)$ \\
\hline \multicolumn{5}{|l|}{ Age (years) } \\
\hline Median & 64.0 & 69.0 & 65.0 & 66.0 \\
\hline Range & $48-73$ & $51-75$ & $63-73$ & $48-75$ \\
\hline \multicolumn{5}{|l|}{ ECOG PS } \\
\hline 0 & 2 & 3 & 5 & $10(62.5)$ \\
\hline 1 & 1 & 3 & 2 & $6(37.5)$ \\
\hline \multicolumn{5}{|l|}{ Prior gastrectomy } \\
\hline Yes & 0 & 0 & 1 & $1(6.3)$ \\
\hline No & 3 & 6 & 6 & $15(93.8)$ \\
\hline \multicolumn{5}{|l|}{ Metastatic sites } \\
\hline Lung & 2 & 2 & 0 & $4(25.0)$ \\
\hline Liver & 1 & 3 & 2 & $6(37.5)$ \\
\hline Lymph nodes & 3 & 6 & 5 & $14(87.5)$ \\
\hline Peritoneum & 1 & 2 & 4 & $7(43.8)$ \\
\hline
\end{tabular}

\section{DLT, MTD, and RD}

Enrollment of patients was started at level 1a, and the three patients at this starting dose level experienced no DLT. Enrollment at level $2 \mathrm{a}$ was started in accordance with the specified procedure for progression to the next level. At level $2 \mathrm{a}$, one of three enrolled patients experienced treatment-related adverse events corresponding to DLT. These treatment-related adverse events were grade 3 fatigue, grade 4 hyperkalemia, and grade 3 anorexia. An additional three patients were enrolled at level $2 \mathrm{a}$, but none experienced treatment-related adverse events corresponding to DLT, thus leading to progression to level 3a. At level 3a, one of three enrolled patients discontinued use of S-1 without permission and was considered ineligible for the DLT evaluation. Therefore, an additional patient was enrolled. None of the three patients included in the DLT evaluation experienced treatment-related adverse events corresponding to DLT, leading to the enrollment of an additional three patients in accordance with the specified procedure. None of these three patients experienced treatment-related adverse events corresponding to DLT. As a result, the MTD was not obtained, and the RD was determined to be level 3a. Consequently, schedule B was not implemented. 


\section{Treatment administered and safety}

The 16 enrolled patients received a total of 102 cycles of study treatment, with a median of 6 cycles (range, 1-18). Adverse events leading to $n a b$-paclitaxel dose reduction were peripheral sensory neuropathy in 7 patients $(43.8 \%)$, neutropenia in 2 patients $(12.5 \%)$, and febrile neutropenia in 1 patient $(6.3 \%)$. Adverse events leading to S-1 dose reduction were neutropenia in 2 patients $(12.5 \%)$, febrile neutropenia in 1 patient $(6.3 \%)$, and anorexia in 1 patient $(6.3 \%)$. Four patients needed dose reduction to the -2 level, all because of peripheral sensory neuropathy. Start of treatment was delayed in 5 patients because of the following adverse events: neutropenia in 2 patients $(12.5 \%)$; fatigue in 1 patient $(6.3 \%)$; nausea in 1 patient $(6.3 \%)$; and anorexia in 1 patient $(6.3 \%)$. Treatment was discontinued for the following reasons: disease progression in 6 patients; adverse events in 9 patients; and completion of the study in 1 patient. Adverse events leading to discontinuation of treatment were peripheral sensory neuropathy $(n=6)$, fatigue $(n=1)$, anorexia $(n=1)$, hyperkalemia $(n=1)$, infectious enteritis $(n=1)$, sepsis $(n=1)$, pyloric stenosis $(n=1)$, and hip fracture $(n=1)$. Treatmentrelated adverse events reported in at least $25 \%$ of all patients are listed in Table 3. Common treatment-related adverse events were neutropenia $(87.5 \% ; n=14)$, leucopenia $(87.5 \% ; n=14)$, peripheral sensory neuropathy
(93.8 \%; $n=15)$, alopecia $(93.8 \% ; n=15)$, and skin hyperpigmentation $(68.8 \% ; n=11)$. Common grade 3 or higher treatment-related adverse events were neutropenia $(62.5 \% ; n=10)$, peripheral sensory neuropathy $(37.5 \%$; $n=6)$, and anorexia $(31.3 \% ; n=5)$. Serious adverse events were reported in 8 patients $(50.0 \%)$. Regarding these adverse events, those reported in 5 patients were assessed as reasonably related to treatment, including febrile neutropenia, peripheral sensory neuropathy, hyperkalemia, fatigue, anorexia, and obstruction gastric. There were no treatment-related deaths during the study.

\section{Efficacy}

For 11 patients with measurable lesion(s) defined by RECIST $\mathrm{v} 1.1$, the response rates are shown in Table 4. No patients had complete response (CR), 6 patients had partial response (PR), 3 patients had stable disease (SD), and 2 patients had progressive disease (PD), with an overall response rate (ORR) of $54.5 \%$ (95\% CI, 23.4-83.3). The median progression-free survival (PFS) in all patients was 5.8 months (95\% CI, 4.3; not reached).

\section{Pharmacokinetics}

A total of nine patients, including three patients at level 1a, three patients at level $2 \mathrm{a}$, and three patients at level $3 \mathrm{a}$,

Table 3 Treatment-related adverse events at each level

\begin{tabular}{|c|c|c|c|c|c|c|c|c|c|c|}
\hline \multirow[t]{2}{*}{ CTCAE term } & \multicolumn{2}{|c|}{ Level 1a $(n=3)$} & \multicolumn{2}{|c|}{ Level 2a $(n=6)$} & \multicolumn{2}{|c|}{ Level 3a $(n=7)$} & \multicolumn{4}{|c|}{ Total $(n=16)$} \\
\hline & All & $\geq$ Grade 3 & All & $\geq$ Grade 3 & All & $\geq$ Grade 3 & All & $(\%)$ & $\geq$ Grade 3 & $(\%)$ \\
\hline Anemia & 1 & 0 & 3 & 0 & 0 & 0 & 4 & $(25.0)$ & 0 & $(0.0)$ \\
\hline Leucopenia & 3 & 1 & 4 & 1 & 7 & 1 & 14 & $(87.5)$ & 3 & $(18.8)$ \\
\hline Neutropenia & 3 & 2 & 4 & 2 & 7 & 6 & 14 & $(87.5)$ & 10 & $(62.5)$ \\
\hline Lymphopenia & 1 & 1 & 3 & 1 & 0 & 0 & 4 & $(25.0)$ & 2 & $(12.5)$ \\
\hline Diarrhea & 0 & 0 & 4 & 1 & 2 & 0 & 6 & $(37.5)$ & 1 & $(6.3)$ \\
\hline Nausea & 1 & 0 & 2 & 0 & 3 & 0 & 6 & $(37.5)$ & 0 & $(0.0)$ \\
\hline Mucositis oral & 1 & 0 & 1 & 1 & 4 & 0 & 6 & $(37.5)$ & 1 & $(6.3)$ \\
\hline Vomiting & 1 & 0 & 2 & 0 & 4 & 0 & 7 & $(43.8)$ & 0 & $(0.0)$ \\
\hline Fatigue & 3 & 0 & 1 & 1 & 3 & 0 & 7 & $(43.8)$ & 1 & $(6.3)$ \\
\hline Anorexia & 3 & 1 & 3 & 2 & 3 & 2 & 9 & $(56.3)$ & 5 & (31.3) \\
\hline Weight loss & 2 & 1 & 2 & 1 & 4 & 1 & 8 & $(50.0)$ & 3 & (18.8) \\
\hline Arthralgia & 1 & 0 & 1 & 0 & 4 & 0 & 6 & $(37.5)$ & 0 & $(0.0)$ \\
\hline Myalgia & 2 & 0 & 1 & 0 & 5 & 0 & 8 & $(50.0)$ & 0 & $(0.0)$ \\
\hline Dysgeusia & 2 & - & 3 & - & 4 & - & 9 & $(56.3)$ & - & - \\
\hline Peripheral sensory neuropathy & 3 & 1 & 5 & 1 & 7 & 4 & 15 & $(93.8)$ & 6 & $(37.5)$ \\
\hline Alopecia & 3 & - & 6 & - & 6 & - & 15 & $(93.8)$ & - & - \\
\hline Rash, maculopapular & 1 & 0 & 2 & 0 & 2 & 0 & 5 & $(31.3)$ & 0 & $(0.0)$ \\
\hline Skin hyperpigmentation & 3 & - & 3 & - & 5 & - & 11 & $(68.8)$ & - & - \\
\hline
\end{tabular}


Table 4 Efficacy of combination therapy according to RECIST v1.1

\begin{tabular}{lllll}
\hline Dose level & Level 1a $(n=2)$ & Level 2a $(n=6)$ & Level 3a $(n=3)$ & Total $(n=11)$ \\
\hline Complete response (CR) & 0 & 0 & 0 & 0 \\
Partial response (PR) & 1 & 3 & 2 & 6 \\
Stable disease (SD) & 1 & 1 & 1 & 3 \\
Progressive disease (PD) & 0 & 2 & 0 & 2 \\
Response rate (CR + RR) (\%) & 50.0 & 50.0 & 66.7 & 54.5 \\
$95 \%$ CI (\%) & $1.3-98.7$ & $11.8-88.2$ & $9.4-99.2$ & $23.4-83.3$ \\
\hline
\end{tabular}

Table 5 Plasma pharmacokinetic parameters for the S-1 components and paclitaxel

\begin{tabular}{|c|c|c|c|c|c|c|c|c|c|c|}
\hline \multirow[t]{2}{*}{ Dose level } & \multicolumn{2}{|l|}{ FT } & \multicolumn{2}{|l|}{$5-\mathrm{FU}$} & \multicolumn{2}{|l|}{ CDHP } & \multicolumn{2}{|l|}{ Oxo } & \multicolumn{2}{|c|}{ Paclitaxel } \\
\hline & Mean & SD & Mean & SD & Mean & SD & Mean & SD & Mean & SD \\
\hline \multicolumn{11}{|l|}{ Level 1a $(n=3)$} \\
\hline$C_{\max }(\mathrm{ng} / \mathrm{ml})$ & 1770 & 463 & 194 & 72 & 302.8 & 170.2 & 42.77 & 28.45 & 8,957 & 933 \\
\hline$t_{\max }(\mathrm{h})$ & 1.3 & 0.6 & 2 & 0 & 1.7 & 0.6 & 2.3 & 1.5 & 0.5 & 0 \\
\hline $\operatorname{AUC}_{0-12}(\mathrm{ng} / \mathrm{h} / \mathrm{ml})$ & 11,770 & 1,990 & 1,014 & 373 & 1,254 & 519 & 200.6 & 110.5 & $8,464^{\mathrm{a}}$ & 578 \\
\hline $\mathrm{AUC}_{\mathrm{inf}}(\mathrm{ng} / \mathrm{h} / \mathrm{ml})$ & 17,340 & 3,030 & 1,027 & 377 & 1,345 & 543 & 300.3 & - & 8,668 & 541 \\
\hline$t_{1 / 2}(\mathrm{~h})$ & 7.2 & 0.5 & 1.6 & 0 & 3 & 0.2 & 4.3 & - & 20.9 & 2.6 \\
\hline \multicolumn{11}{|l|}{ Level $2 \mathrm{a}(n=3)$} \\
\hline$C_{\max }(\mathrm{ng} / \mathrm{ml})$ & 2107 & 596 & 162.6 & 59.1 & 331 & 162 & 59.74 & 46.06 & 10,760 & 1,350 \\
\hline$t_{\max }(\mathrm{h})$ & 2 & 0 & 3.3 & 1.2 & 2 & 0 & 2 & 0 & 0.5 & 0 \\
\hline $\operatorname{AUC}_{0-12}(\mathrm{ng} / \mathrm{h} / \mathrm{ml})$ & 16,100 & 3,610 & 865 & 249.9 & 1,344 & 352 & 244.1 & 162.5 & $14,930^{\mathrm{a}}$ & 5,460 \\
\hline $\mathrm{AUC}_{\text {inf }}(\mathrm{ng} / \mathrm{h} / \mathrm{ml})$ & 27,260 & 8,870 & 881.8 & 242.5 & 1,416 & 334 & 351.5 & - & 15,210 & 5,470 \\
\hline$t_{1 / 2}(\mathrm{~h})$ & 8.7 & 3.1 & 1.7 & 0.3 & 2.7 & 0.4 & 3.4 & - & 19.5 & 2.5 \\
\hline \multicolumn{11}{|l|}{ Level 3a $(n=3)$} \\
\hline$C_{\max }(\mathrm{ng} / \mathrm{ml})$ & 2139 & 180 & 146.2 & 57.9 & 309.3 & 19.9 & 86.59 & 38.17 & 11,100 & 1,130 \\
\hline$t_{\max }(\mathrm{h})$ & 1.3 & 0.6 & 2.7 & 1.2 & 1.3 & 0.6 & 1.7 & 0.6 & 0.5 & 0 \\
\hline $\operatorname{AUC}_{0-12}(\mathrm{ng} / \mathrm{h} / \mathrm{ml})$ & 14,100 & 2,810 & 794.8 & 273.3 & 1,345 & 154 & 324.2 & 87.5 & $12,760^{\mathrm{a}}$ & 1,500 \\
\hline $\mathrm{AUC}_{\mathrm{inf}}(\mathrm{ng} / \mathrm{h} / \mathrm{ml})$ & 24,230 & 7,050 & 813.2 & 271.7 & 1,462 & 155 & 351.8 & 82.9 & 12,990 & 1,470 \\
\hline$t_{1 / 2}(\mathrm{~h})$ & 9.9 & 1.4 & 1.9 & 0.2 & 3.5 & 0.7 & 2.7 & 1.3 & 19.4 & 5.6 \\
\hline
\end{tabular}

$C_{\max }$ maximum plasma concentration, $t_{\max }$ time to $C_{\max }, A U C$ area under the concentration-time curve, $A U C_{0-12}$ AUC from 0 to $12 \mathrm{~h}, A U C_{i n f}$ AUC from 0 to infinity, $t_{1 / 2}$ terminal elimination half-life

a $\mathrm{AUC}_{0-72}$ were included in the pharmacokinetic evaluation. The pharmacokinetic parameters of the S-1 components and paclitaxel are summarized in Table 5. As S-1 was administered at a fixed dose during the different levels of combination therapy, there were no differences in any parameters for the S-1-derived FT, 5-FU, CDHP, and Oxo among the levels after administration of S-1 in combination with nab-paclitaxel. Also, the pharmacokinetic parameters of S-1 and nab-paclitaxel in this study were almost comparable to those of each compound in previous studies of S-1 monotherapy [11-13] or nab-paclitaxel monotherapy [14].

\section{Discussion}

In this study, the dose level was escalated up to level 3a, but the MTD was not obtained. However, the RD for combination therapy was determined to be $80 \mathrm{mg} / \mathrm{m}^{2}$ for $\mathrm{S}-1$ and $260 \mathrm{mg} / \mathrm{m}^{2}$ for $n a b$-paclitaxel, a combination of the RD of each drug administered alone. The median relative dose intensity (RDI) of nab-paclitaxel and S-1 during the first two cycles was $97.5 \%$ and $87.20 \%$ at level 1a, $97.50 \%$ and $95.95 \%$ at level $2 \mathrm{a}$, and $100.00 \%$ and $73.20 \%$ at level 3a, respectively. The RDI of S-1 at level 3a was lower because one patient at this level suspended S-1 without permission; however, the treatment 
compliance did not differ among the dose levels in patients who were evaluated for the DLT, excluding this patient.

The only treatment-related adverse event that occurred more frequently after coadministration of S-1 and nabpaclitaxel, compared with either drug alone [5, 10], was neutropenia. Specifically, the incidence of grade 3 or higher treatment-related neutropenia was $62.5 \% \quad(10$ patients) in the present study, compared with $11 \%$ (16 patients) for S-1 alone [5] and 49.1\% (27 patients) for $n a b$ PTX alone [10], suggesting that our combination therapy may be associated with increased myelosuppression.

Neutropenia required prolonged washout (delay in the start of the next cycle) in only 2 patients at level $3 \mathrm{a}$ $(12.5 \%)$ among the 16 patients. Neutropenia led to suspension of S-1 in 7 (43.8\%) of 16 patients, comprising 2 patients $(66.7 \%)$ at level 1a, 1 patient $(16.7 \%)$ at level 2a, and 4 patients $(57.1 \%)$ at level 3a. Neutropenia quite often led to suspension of $\mathrm{S}-1$, but progressed to febrile neutropenia in only 1 patient at level $1 \mathrm{a}$. In addition, neutropenia required G-CSF therapy in only 2 of 16 patients and could be managed by suspension in many patients, indicating that this adverse event can be controlled with appropriate treatment.

Peripheral sensory neuropathy, the most common adverse event related to nab-paclitaxel, was reported in 15 $(93.8 \%)$ of 16 patients. Grade 3 or higher peripheral sensory neuropathy was reported in $6(37.5 \%)$ of 16 patients. The incidence of peripheral sensory neuropathy following administration of $n a b$-paclitaxel alone [10] was $92.7 \%$ for all grades and $23.6 \%$ for grade 3 or higher, indicating that the incidence did not differ greatly between the combination therapy and nab-paclitaxel monotherapy. In the present study, peripheral sensory neuropathy led to dose reduction in $7(43.8 \%)$ of 16 patients and $6(85.7 \%)$ of 7 patients at level 3a, which was identified as the RD. In addition, peripheral sensory neuropathy led to discontinuation of treatment in 6 of 16 patients ( 2 patients at level 1a, 1 patient at level $2 \mathrm{a}$, and 3 patients at level $3 \mathrm{a}$ ), indicating that timely suspension and dose reduction of $n a b$-PTX may be necessary to continue combination therapy for a longer period of time.

Other treatment-related adverse events did not occur more frequently after the combination therapy compared with $n a b$-paclitaxel or S-1 monotherapy.

The pharmacokinetic parameters of S-1 and nab-paclitaxel calculated in this study were compared with those of each compound calculated in previous studies of $\mathrm{S}-1$ monotherapy [11-13] or nab-paclitaxel monotherapy [14] to evaluate the pharmacokinetics of the two compounds upon coadministration. There were no differences in any of the pharmacokinetic parameters of FT, 5-FU, CDHP, Oxo, or paclitaxel, showing that the pharmacokinetics of S-1 or paclitaxel following $\mathrm{S}-1 / n a b$-PTX combination therapy for unresectable or recurrent gastric cancer did not differ greatly from the pharmacokinetics of each compound administered alone.

Although SP therapy is a standard first-line treatment for gastric cancer, the present study, despite involving only a small number of patients, showed an ORR of $54.5 \%$ (95 \% CI, 23.4-83.3) in patients with measurable lesion(s) defined by RECIST v1.1 and a median PFS of 5.8 months (95\% CI 4.3, not reached) in 16 patients. These outcomes were comparable to the ORR of $54 \%$ and PFS of 6.0 months following SP therapy in the SPIRITS trial [5], indicating that the S-1/nab-paclitaxel combination therapy can be expected to be as effective as SP therapy. The S-1/nab-paclitaxel combination therapy is also expected to be indicated for patients in whom platinum cannot be used, more specifically, those for whom CDDP is contraindicated, including elderly patients and patients with severe peritoneal stenosis. Furthermore, because the RD for this combination therapy was determined to be the maximum dose of each drug, and the RDI during the first two cycles was acceptable, the combination therapy can be expected to be applied as a preoperative chemotherapy, which is administered on a short-term basis.

While our study was being conducted on a first-line treatment for AGC, the results from the WJOG4007 trial [15] and RAINBOW trial [16] on second-line treatments for AGC were published. The WJOG4007 trial failed to demonstrate the superiority of CPT-11 to weekly paclitaxel in terms of OS. The RAINBOW trial demonstrated the superiority of combination therapy with paclitaxel and ramucirumab to weekly paclitaxel in terms of overall survival (OS). Given these results, paclitaxel was established as a second-line treatment. Currently, the noninferiority of triweekly $\left(260 \mathrm{mg} / \mathrm{m}^{2}\right)$ or weekly $\left(100 \mathrm{mg} / \mathrm{m}^{2}\right) \mathrm{nab}$-paclitaxel to weekly paclitaxel used as a control arm in terms of OS is being evaluated to establish the appropriateness of $n a b$-paclitaxel as a second-line treatment for AGC in the ABSOLUTE trial [17].

The outcomes of a combination therapy with weekly $n a b$-paclitaxel and S-1 were reported in a phase I study on metastatic breast cancer [18]. The RD was $100 \mathrm{mg} / \mathrm{m}^{2}$ weekly for $n a b$-paclitaxel and $80 \mathrm{mg} / \mathrm{m}^{2}$ daily for 2 weeks in each 3-week cycle for S-1. The incidence of grade 3 or higher peripheral sensory neuropathy was $13.3 \%$ (2 of 15 patients), which was lower than the incidence of $37.5 \%$ after the combination therapy in the present study. Although weekly $n a b$-paclitaxel is expected to reduce treatment-related adverse events, combination therapy with weekly $n a b$-paclitaxel and $\mathrm{S}-1$ for gastric cancer is expected to be developed if $n a b$-paclitaxel at $100 \mathrm{mg} / \mathrm{m}^{2}$ weekly is established by the ABSOLUTE trial (currently in progress). 
In conclusion, the $\mathrm{S}-1 / n a b$-paclitaxel combination therapy evaluated in the present phase I study raised no major safety concerns, although hematotoxicity was enhanced compared with monotherapy with either drug, thereby showing that the combination therapy is a potential new non-platinum-based treatment regimen for unresectable or recurrent gastric cancer. The long-term safety and efficacy of this combination therapy will be evaluated in phase II studies.

Acknowledgments We thank all the participating patients and their families, as well as the investigators and clinical research coordinators. We are grateful to Yutaka Ariyoshi, Keisuke Aiba, and Hideo Baba, who served as members of the Data and Safety Monitoring Committee, and Atsushi Sato, who served as medical officer. This study was sponsored by and designed under the responsibility of Taiho Pharmaceutical Co., Ltd. Study drug was provided by Taiho Pharmaceutical Co., Ltd.

\section{Compliance with ethical standards}

Conflict of interest The authors declare that they have no conflict of interest.

Human rights statement and informed consent All procedures followed were in accordance with the ethical standards of the responsible committee on human experimentation (institutional and national) and with the Helsinki Declaration of 1964 and later versions. Informed consent or substitute for it was obtained from all patients for being included in the study.

\section{References}

1. Ferlay J, Soerjomataram I, Dikshit R, Eser S, Mathers C, Rebelo $\mathrm{M}$, et al. Cancer incidence and mortality worldwide: sources, methods and major patterns in GLOBOCAN 2012. Int J Cancer. 2015;136:E359-86.

2. Ohtsu A, Shimada Y, Shirao K, Boku N, Hyodo I, Saito H, et al. Randomized phase III trial of fluorouracil alone versus fluorouracil plus cisplatin versus uracil and tegafur plus mitomycin in patients with unresectable, advanced gastric cancer: the Japan Clinical Oncology Group Study (JCOG9205). J Clin Oncol. 2003;21(1):54-9.

3. Shirasaka T, Shimamato Y, Ohshimo H, Yamaguchi M, Kato T, Yonekura $\mathrm{K}$, et al. Development of a novel form of an oral 5-fluorouracil derivative (S-1) directed to the potentiation of the tumor selective cytotoxicity of 5 -fluorouracil by two biochemical modulators. Anticancer Drugs. 1996;7(5):548-57.

4. Boku N, Yamamoto S, Fukuda H, Shirao K, Doi T, Sawaki A, et al. Fluorouracil versus combination of irinotecan plus cisplatin versus S-1 in metastatic gastric cancer: a randomised phase 3 study. Lancet Oncol. 2009;10(11):1063-9.

5. Koizumi W, Narahara H, Hara T, Takagane A, Akiya T, Takagi $\mathrm{M}$, et al. S-1 plus cisplatin versus S-1 alone for first-line treatment of advanced gastric cancer (SPIRITS trial): a phase III trial. Lancet Oncol. 2008;9(3):215-21.
6. Nukatsuka M, Fujioka A, Nakagawa F, Oshimo H, Kitazato K, Uchida J, et al. Antimetastatic and anticancer activity of S-1, a new oral dihydropyrimidine-dehydrogenase-inhibiting fluoropyrimidine, alone and in combination with paclitaxel in an orthotopically implanted human breast cancer model. Int $\mathrm{J}$ Oncol. 2004;25(6):1531-6.

7. Fujitani K, Narahara H, Takiuchi H, Tsujinaka T, Satomi E, Gotoh M, et al. Phase I and pharmacokinetic study of S-1 combined with weekly paclitaxel in patients with advanced gastric cancer. Oncology. 2005;69(5):414-20.

8. Mochiki E, Ohno T, Kamiyama Y, Aihara R, Haga N, Ojima H, et al. Phase I/II study of S-1 combined with paclitaxel in patients with unresectable and/or recurrent advanced gastric cancer. $\mathrm{Br} \mathrm{J}$ Cancer. 2006;95(12):1642-7.

9. Hokita S, Aikou T, Miyazono F, Ishigami S, Aridome K, Maenohara S, et al. A phase I combination chemotherapy study of biweekly paclitaxel and S-1 administration in patients with advanced gastric cancer. Cancer Chemother Pharmacol. 2006;57(6):736-40.

10. Sasaki Y, Nishina T, Yasui H, Goto M, Muro K, Tsuji A, et al. Phase II trial of nanoparticle albumin-bound paclitaxel as secondline chemotherapy for unresectable or recurrent gastric cancer. Cancer Sci. 2014;105(7):812-7.

11. Furuse J, Okusaka T, Kaneko S, Kudo M, Nakachi K, Ueno H, et al. Phase I/II study of the pharmacokinetics, safety and efficacy of S-1 in patients with advanced hepatocellular carcinoma. Cancer Sci. 2010;101(12):2606-11.

12. Ueno H, Okusaka T, Ikeda M, Takezako Y, Morizane C. An early phase II study of S-1 in patients with metastatic pancreatic cancer. Oncology. 2005;68(2-3):171-8.

13. Ueno H, Okusaka T, Ikeda M, Takezako Y, Morizane C. Phase II study of S-1 in patients with advanced biliary tract cancer. Br J Cancer. 2004;91(10): 1769-74.

14. Yamada K, Yamamoto N, Yamada Y, Mukohara T, Manami H, Tamura T. Phase I and pharmacokinetic study of ABI-007, albumin-bound paclitaxel, administered every 3 weeks in Japanese patients with solid tumors. Jpn J Clin Oncol. 2010;40(5):404-11.

15. Hironaka S, Ueda S, Yasui $\mathrm{H}$, Nishina T, Tsuda M, Tsumura T, et al. Randomized, open-label, phase III study comparing irinotecan with paclitaxel in patients with advanced gastric cancer without severe peritoneal metastasis after failure of prior combination chemotherapy using fluoropyrimidine plus platinum: WJOG 4007 trial. J Clin Oncol. 2013;31(35):4438-44.

16. Wilke H, Muro K, Cutsem EV, Oh SC, Bodoky G, Shimada Y, et al. Ramucirumab plus paclitaxel versus placebo plus paclitaxel in patients with previously treated advanced gastric or gastrooesophageal junction adenocarcinoma (RAINBOW): a doubleblind, randomised phase 3 trial. Lancet Oncol. 2014;15(11):1224-35.

17. Koizumi W, Morita S, Sakata Y. A randomized phase III trial of weekly or 3-weekly doses of nab-paclitaxel versus weekly doses of Cremophor-based paclitaxel in patients with previously treated advanced gastric cancer (ABSOLUTE trial). Jpn J Clin Oncol. 2014;45(3):303-6.

18. Tsurutani J, Kuroi K, Iwasa T, Miyazaki M, Nishina S, Makimura $\mathrm{C}$, et al. Phase I study of weekly nab-paclitaxel combined with $\mathrm{S}-1$ in patients with human epidermal growth factor receptor type 2-negative metastatic breast cancer. Cancer Sci. 2015;106(6):734-9. 